\title{
PERSPECTIVA DEL DERECHO DEL MEDIO AMBIENTE Y DE LAS POLÍTICAS AMBIENTALES DE LA UNIÓN EUROPEA
}

(SEGUNDO SEMESTRE 2019)

\author{
Pol Pallàs Secall \\ Investigador predoctoral FPI \\ Universitat de Barcelona
}


Sumario: 1. Introducción. 2. Actos jurídicos en el ámbito de la política europea del medio ambiente. 2.1. Actos legislativos. a) Reglamentos. b) Directivas. c) Decisiones. 2.2. Actos delegados. a) Reglamentos delegados. b) Decisiones delegadas. 2.3. Actos de ejecución. a) Reglamentos de ejecución. b) Decisiones de ejecución. 2.4. Acuerdos internacionales y posiciones en organismos internacionales. 3. Otras actividades y actos en el ámbito de la política europea del medio ambiente. 3.1 Consultas públicas. 3.2. Resoluciones no legislativas del Parlamento Europeo. 3.3. Informes y comunicaciones de la Comisión Europea. 3.4. Comunicaciones conjuntas de la Comisión Europea y el Alto Representante. 3.5. Dictámenes del Comité de las Regiones y del Comité Económico y Social Europeo. 4. Procedimientos de infracción.

\section{INTRODUCCIÓN}

Esta crónica comprende el periodo que va del 1 de marzo de 2019 al 30 de septiembre de 2019. En ella se recoge toda la legislación medioambiental de las instituciones de la Unión Europea aprobada en este lapso de tiempo, ya sea la legislación contra el cambio climático, la protección de la biodiversidad o la legislación sobre contaminantes, pero también la legislación en otros ámbitos que pueden tener afectación en el medioambiente, como son la energía o la política pesquera.

A parte de los actos jurídicos, también se exponen otras iniciativas y actividades de la política europea del medioambiente que han tenido lugar durante este periodo, como son las consultas públicas de la Comisión y sus comunicaciones e informes, las resoluciones no legislativas del Parlamento Europeo o los dictámenes del Comité Europeo de las Regiones y del Comité Económico y Social Europeo. Por último, se exponen los procedimientos de infracción contra los Estados miembros de la Unión iniciados por la Comisión por infracciones de la normativa medioambiental europea.

Destacan en este periodo las numerosas normas aprobadas en el ámbito del transporte para la reducción de las emisiones de $\mathrm{CO}^{2}$ de los vehículos nuevos con el objetivo de cumplir los objetivos para 2030. En el ámbito energético se aprobaron un Reglamento y una Directiva que liberalizan el mercado eléctrico y facilitan el acceso a la red de la energía proveniente de fuentes renovables. También se aprobó una Directiva para la prevención del abandono de plásticos de un solo uso en el medioambiente mediante la promoción de la economía circular. 
Como ya se pudo ver en anteriores ediciones, durante el periodo de tiempo comprendido en esta crónica también se han aprobado diversas normas y actos en el ámbito del medio ambiente motivados por la salida de la Unión por pate de Reino Unido. Como el lector verá, responden al esfuerzo de la UE para disminuir los efectos adversos de dicha salida.

Aunque no será de mayor comentario en esta crónica, cabe mencionar que en marzo de 2019 se llegó a un acuerdo provisional entre el Parlamento Europeo y el Consejo sobre el programa LIFE para el Medioambiente y la Acción Climática para el próximo Marco Financiero Plurianual 2021-2027. Este queda pendiente ahora de su aprobación formal.

\section{ACTOS JURÍDICOS EN EL ÁMBITO DE LA POLÍTICA EUROPEA DEL MEDIO AMBIENTE.}

\subsection{Actos legislativos}

\section{a) Reglamentos}

Durante el periodo analizado en esta crónica se han aprobado dos reglamentos referentes a la limitación de las emisiones de $\mathrm{CO}^{2}$ por parte de vehículos nuevos, tanto de turismos y vehículos comerciales ligeros, como de vehículos pesados: - Reglamento (UE) 2019/631 del Parlamento Europeo y del Consejo, de 17 de abril de 2019, por el que se establecen normas de comportamiento en materia de emisiones de $\mathrm{CO}^{2}$ de los turismos nuevos y de los vehículos comerciales ligeros nuevos, y por el que se derogan los Reglamentos (CE) n. ${ }^{\circ}$ 443/2009 y (UE) n. ${ }^{\circ}$ 510/2011 - Reglamento (UE) 2019/1242 del Parlamento Europeo y del Consejo, de 20 de junio de 2019, por el que se establecen normas de comportamiento en materia de emisiones de $\mathrm{CO}^{2}$ para vehículos pesados nuevos y se modifican los Reglamentos (CE) 595/2009 y (UE) 2018/956 del Parlamento Europeo y del Consejo y la Directiva 96/53/CE del Consejo. Estas dos normas se encuadran en el trabajo de la Unión Europea por aplicar los objetivos establecidos en el Acuerdo de París de 2015, entre los cuales se incluye evitar que la temperatura media mundial rebase los $2^{\circ} \mathrm{C}$ de los niveles preindustriales. Son una medida acorde con el Reglamento (UE) 2018/842 del Parlamento Europeo y del Consejo que obliga a los sectores no 
incluidos en el comercio de derechos de emisión de la UE a la reducción de un $30 \%$ de las emisiones de gases de efecto invernadero para 2030 en relación a las emisiones de 2005. Así mismo, estos dos reglamentos dan seguimiento a la Comunicación de la Comisión de 28 de noviembre de 2018 "Un planeta limpio para todos: una visión estratégica europea a largo plazo para una economía próspera, moderna, competitiva y climáticamente neutra" -que ya comentamos en la anterior crónica de esta colección-. Dichos reglamentos establecen unos límites de emisiones de gases de efecto invernadero a aplicar a los vehículos nuevos a partir de 2020 de forma gradual y en dos etapas, en 2025 y 2030 . En el ámbito de la energía es relevante para el objeto de esta crónica la aprobación del Reglamento (UE) 2019/943 del Parlamento Europeo y del Consejo, de 5 de junio de 2019, relativo al mercado interior de la electricidad, que se aprobó junto a la Directiva (UE) 2019/944 del Parlamento Europeo y del Consejo, de 5 de junio de 2019, sobre normas comunes para el mercado interior de la electricidad y por la que se modifica la Directiva 2012/27/UE. Dicho reglamento es una versión refundida del Reglamento (CE) 714/2009 del Parlamento Europeo y del Consejo, que ha sido modificado en varias ocasiones. Asienta los principios regulatorios por lo que respecta a la operación en los mercados de la energía eléctrica, permitiendo la libre formación de precios en función de la oferta y la demanda, así como permitiendo a los consumidores participar en el mercado de la energía. El Reglamento establece las normas para el comercio transfronterizo de energía eléctrica y los principios para la fijación de las tarifas armonizadas de su transporte. Conviene destacar que el sexto principio, enumerado en el artículo 3.f. de este Reglamento y por el que la regulación de los Estados miembros debe facilitar la descarbonización de la energía, no establece una priorización de la energía procedente de fuentes renovables, sino que se limita a "permitir" integrar dicha energía en el mercado. Aún en el ámbito de la energía, se aprobó el Reglamento (UE) 2019/424 de la Comisión, de 15 de marzo de 2019, por el que se establecen requisitos de diseño ecológico para servidores y productos de almacenamiento de datos de conformidad con la Directiva 2009/125/CE del Parlamento Europeo y del Consejo, y por el que se modifica el Reglamento (UE) n. ${ }^{\circ}$ 617/2013 de la Comisión. Este reglamento regula los requisitos ecológicos para la entrada en el mercado de servidores informáticos y productos de almacenamiento en 
línea. Se trata de una regulación diferenciada y excluyente respecto a otros aparatos informáticos regulados en el Reglamento (UE) $n{ }^{\circ}$ 617/2013 de la Comisión, de 26 de junio de 2013, por el que se desarrolla la Directiva 2009/125/CE del Parlamento Europeo y del Consejo en lo relativo a los requisitos de diseño ecológico aplicables a los ordenadores y servidores informáticos. En el ámbito del registro, evaluación, autorización y restricción de sustancias y mezclas químicas (REACH) se han aprobado dos reglamentos durante el periodo analizado: - Reglamento (UE) 2019/957 de la Comisión, de 11 de junio de 2019, que modifica, por lo que respecta al (3,3,4,4,5,5,6,6,7,7,8,8,8-tridecafluorooctil) silanetriol y sus TDFA, el anexo XVII del Reglamento (CE) n. ${ }^{\circ}$ 1907/2006 del Parlamento Europeo y del Consejo, relativo al registro, la evaluación, la autorización y la restricción de las sustancias y mezclas químicas (REACH), establece una restricción al uso y comercialización de la sustancia que figura en su título. - Reglamento (UE) 2019/1390 de la Comisión, de 31 de julio de 2019, que modifica, con vistas a su adaptación al progreso técnico, el anexo del Reglamento (CE) 440/2008, por el que se establecen métodos de ensayo de acuerdo con el Reglamento (CE) 1907/2006 del Parlamento Europeo y del Consejo, relativo al registro, la evaluación, la autorización y la restricción de las sustancias y mezclas químicas (REACH). Este adapta la normativa REACH a las directrices de ensayo y uso de animales de la OCDE para la determinación de la ecotoxicidad y la peligrosidad para la salud humana de las sustancias químicas. En materia de contaminantes orgánicos se aprobó el Reglamento (UE) 2019/1021 del Parlamento Europeo y del Consejo, de 20 de junio de 2019, sobre contaminantes orgánicos persistentes. Este refunde las modificaciones hechas hasta la fecha del Reglamento (CE) no 850/2004 del Parlamento Europeo y del Consejo, de 29 de abril de 2004, sobre contaminantes orgánicos persistentes y por el que se modifica la Directiva 79/117/CE. En el ámbito de la gobernanza medioambiental de la Unión se aprobó el Reglamento (UE) 2019/1010 del Parlamento Europeo y del Consejo, de 5 de junio de 2019, relativo a la adaptación de las obligaciones de información en el ámbito de la legislación relativa al medio ambiente y por el que se modifican los Reglamentos (CE) 166/2006 y (UE) 995/2010 del Parlamento Europeo y del Consejo, las Directivas 2002/49/CE, 2004/35/CE, 2007/2/CE, 2009/147/CE y 2010/63/UE 
del Parlamento Europeo y del Consejo, los Reglamentos (CE) 338/97 y (CE) 2173/2005 del Consejo, y la Directiva 86/278/CEE del Consejo. Tiene por objetivo reducir la carga administrativa derivada de la obligación de información de los actores implicados en la gestión del medioambiente, ya sean ONG o empresas. También pretende hacer más fluido el intercambio de información entre entidades públicas y hacer más transparente la gestión medioambiental de los Estados miembros para con la UE. Para tal fin se modifican un conjunto de regulaciones medioambientales que incluyen tres Reglamentos y siete Directivas. Debido a la afectación que tiene sobre el medio marino, debemos abordar también aquí la regulación de la UE referente a la pesca. Por tal de mejorar las medidas técnicas ya en vigor dirigidas a disminuir el número de capturas no deseadas que ponen en riesgo la conservación de las especies marinas, se aprobó el Reglamento (UE) 2019/1241 del Parlamento Europeo y del Consejo, de 20 de junio de 2019, sobre la conservación de los recursos pesqueros y la protección de los ecosistemas marinos con medidas técnicas, y por el que se modifican los Reglamentos (CE) 2019/2006 y (CE) 1224/2009 del Consejo y los Reglamentos (UE) 1380/2013, (UE) 2016/1139, (UE) 2018/973, (UE) 2019/472 y (UE) 2019/1022 del Parlamento Europeo y del Consejo, y por el que se derogan los Reglamentos (CE) 894/97, (CE) 850/98, (CE) 2549/2000, (CE) 254/2002, (CE) 812/2004 y (CE) 2187/2005 del Consejo. También se aprobaron dos Reglamentos para el establecimiento de medidas de conservación y restricciones a la pesca por regiones marítimas de la Unión: Reglamento (UE) 2019/472 del Parlamento Europeo y del Consejo, de 19 de marzo de 2019, por el que se establece un plan plurianual para las poblaciones pescadas en las aguas occidentales y aguas adyacentes, así como para las pesquerías que explotan estas poblaciones, se modifican los Reglamentos (UE) 2016/1139 y (UE) 2018/973 y se derogan los Reglamentos (CE) n. ${ }^{\circ}$ 811/2004, (CE) n. ${ }^{\circ} 2166 / 2005$, (CE) n. ${ }^{\circ} 388 / 2006$, (CE) n. ${ }^{\circ}$ 509/2007 y (CE) n. ${ }^{\circ}$ 1300/2008 del Consejo - Reglamento (UE) 2019/529 del Consejo, de 28 de marzo de 2019, por el que se modifica el Reglamento (UE) 2019/124 en lo que respecta a determinadas posibilidades de pesca - Reglamento (UE) 2019/1022 del Parlamento Europeo y del Consejo, de 20 de junio de 2019, por el que se establece un plan plurianual para la pesca demersal en el Mediterráneo occidental y por el que se modifica el Reglamento (UE) n. ${ }^{\circ}$ 508/2014 - 
Reglamento (UE) 2019/1097 del Consejo, de 26 de junio de 2019, por el que se modifica el Reglamento (UE) 2019/124 en lo que respecta a determinadas posibilidades de pesca - Reglamento (UE) 2019/1154 del Parlamento Europeo y del Consejo, de 20 de junio de 2019, relativo a un plan de recuperación plurianual para el pez espada del Mediterráneo y por el que se modifican el Reglamento (CE) n. ${ }^{\circ}$ 1967/2006 del Consejo y el Reglamento (UE) 2017/2107 del Parlamento Europeo y del Consejo Se aprobaron, asimismo, un conjunto de Reglamentos para la aplicación de medidas derivadas de directivas determinadas por organizaciones regionales del ámbito pesquero: Reglamento (UE) 2019/982 del Parlamento Europeo y del Consejo, de 5 de junio de 2019, por el que se modifica el Reglamento (UE) 1343/2011 sobre determinadas disposiciones aplicables a la pesca en la zona del Acuerdo CGPM (Comisión General de Pesca del Mediterráneo) - Reglamento (UE) 2019/833 del Parlamento Europeo y del Consejo, de 20 de mayo de 2019, por el que se establecen medidas de conservación y ejecución aplicables en la zona de regulación de la Organización de Pesquerías del Atlántico Noroeste, se modifica el Reglamento (UE) 2016/1627 y se derogan los Reglamentos (CE) n. ${ }^{\circ}$ 2115/2005 y (CE) n. ${ }^{\circ} 1386 / 2007$ del Consejo. Se aprobaron tres Reglamentos relativos al reparto entre los Estados miembros de la Unión de posibilidades de pesca derivadas de acuerdos pesqueros con terceros Estados: - Reglamento (UE) 2019/952 del Consejo, de 17 de mayo de 2019, relativo al reparto de las posibilidades de pesca en virtud del Protocolo de aplicación del Acuerdo de Asociación en el Sector Pesquero entre la Comunidad Europea y la República de Cabo Verde (2019-2024) - Reglamento (UE) 2019/1089 del Consejo, de 6 de junio de 2019, relativo al reparto de las posibilidades de pesca en virtud del Protocolo de aplicación del Acuerdo de Colaboración en el Sector Pesquero entre la Comunidad Europea y la República de Guinea-Bisáu (2019-2024) - Reglamento (UE) 2019/1333 del Consejo, de 25 de junio de 2019, sobre el reparto de las posibilidades de pesca en virtud del Protocolo de aplicación del Acuerdo de Colaboración de Pesca Sostenible entre la Unión Europea y la República de Gambia Por último cabe señalar la aprobación de un Reglamento en el ámbito de la política pesquera europea motivado por la futura salida del Reino Unido de la Unión Europea: Reglamento (UE) 2019/498 del Parlamento Europeo y del Consejo, de 25 de 
marzo de 2019, por el que se modifica el Reglamento (UE) 2017/2403 en lo que se refiere a las autorizaciones de pesca para los buques pesqueros de la Unión en aguas del Reino Unido y las operaciones de pesca de los buques pesqueros del Reino Unido en aguas de la Unión. Para evitar que la salida del Reino Unido deje sin posibilidades de pesca a los buques de la Unión en aguas del Reino Unido y viceversa, este Reglamento establece un marco jurídico para el acceso recíproco de los buques de uno y otro territorio.

\section{b) Directivas}

Durante el periodo analizado se han aprobado cinco Directivas que afectan al ámbito del medioambiente. En primer lugar, encontramos la Directiva (UE) 2019/782 de la Comisión, de 15 de mayo de 2019, por la que se modifica la Directiva 2009/128/CE del Parlamento Europeo y del Consejo en lo que respecta al establecimiento de indicadores de riesgo armonizados. Esta Directiva pretende dar continuidad al compromiso de establecer indicadores de riesgo armonizados que la Comisión asumió debido a la Iniciativa Ciudadana Europea de 2017 "Prohibición del glifosato y protección de las personas y del medio ambiente frente a los pesticidas tóxicos". Con los indicadores armonizados establecidos se pretende dotar de herramientas a los Estados miembros y a la Unión para la medición de la reducción de los riesgos derivados del uso de plaguicidas.

En el ámbito de la reducción de la contaminación del medio marino se aprobó la Directiva (UE) 2019/883 del Parlamento Europeo y del Consejo, de 17 de abril de 2019, relativa a las instalaciones portuarias receptoras a efectos de la entrega de desechos generados por buques, por la que se modifica la Directiva 2010/65/UE y se deroga la Directiva 2000/59/CE. La normativa europea sobre la contaminación del medio marino por parte de buques se basa en la aplicación del Convenio internacional para prevenir la contaminación por los buques (Convenio MARPOL), que establece prohibiciones de descarga de residuos en el mar. La Directiva en cuestión se ve motivada por la constatación de que se siguen produciendo descargas ilegales en el mar por parte de buques, para lo que se establecen incentivos para la descarga controlada en los puertos, así como un conjunto de medidas de control. 
Durante el periodo analizado se aprobó también una Directiva sobre los residuos plásticos: Directiva (UE) 2019/904 del Parlamento Europeo y del Consejo, de 5 de junio de 2019, relativa a la reducción del impacto de determinados productos de plástico en el medio ambiente. En ella se establecen una serie de medidas por tal de reducir la producción y el uso de productos plásticos de un solo uso, es decir, sin incluir todos los productos plásticos que tienen más de un uso. Su objetivo es evitar el abandono de este tipo de productos en el medioambiente, en especial el medio marino, para lo que pretende incentivar un modelo de economía circular. La Directiva centra la atención en la reducción del consumo de plástico, pero hay que decir que es poco ambiciosa en la limitación de la introducción de ciertos productos en el mercado. Estos últimos se limitan a los enumerados en la lista de la parte $B$ del anexo y a todos los productos hechos con plásticos oxodegradables. Esta Directiva se suma a las Directivas 94/62/CE y 2008/98/CE sobre residuos y embalaje respectivamente, pero en caso de conflicto entre ellas la primera tiene primacía sobre las segundas por ser una norma especial.

Ya comentamos al principio de esta crónica que el Reglamento (UE) 2019/943 del Parlamento Europeo y del Consejo, de 5 de junio de 2019, relativo al mercado interior de la electricidad venía acompañado de la Directiva (UE) 2019/944 del Parlamento Europeo y del Consejo, de 5 de junio de 2019, sobre normas comunes para el mercado interior de la electricidad y por la que se modifica la Directiva 2012/27/UE. Desde el punto de vista ambiental, la nueva Directiva es relevante tanto en cuanto favorece la conexión de las fuentes de energía renovable a la red eléctrica, incrementa la flexibilidad y adaptabilidad de los distribuidores minoristas de energía, así como la capacidad de los consumidores para escoger fuentes de energía renovable. También se incentiva el desarrollo y uso de nuevas tecnologías que permitan superar la variabilidad de las fuentes de energía renovable y mejoren las capacidades técnicas de almacenamiento de electricidad.

En el ámbito del transporte se aprobó la Directiva (UE) 2019/1161 del Parlamento Europeo y del Consejo, de 20 de junio de 2019, por la que se modifica la Directiva 2009/33/CE relativa a la promoción de vehículos de transporte por carretera limpios y energéticamente eficientes. Esta Directiva 
regula los incentivos al transporte de bajas emisiones de $\mathrm{CO}^{2}$ que deben hacer las administraciones públicas por medio de la contratación pública. Supone una actualización de la Directiva 2009/33/CE y establece cambios a la luz de la poca efectividad de esta última para lograr el objetivo de una mayor aceptación de los vehículos limpios en la Unión.

\section{c) Decisiones}

Durante el periodo de tiempo analizado se aprobaron tres Decisiones en aplicación del Reglamento (CE) 1221/2009 del Parlamento Europeo y del Consejo, de 25 de noviembre de 2009, relativo a la participación voluntaria de organizaciones en un sistema comunitario de gestión y auditoría medioambientales (EMAS). Según este se debía elaborar un "documento de referencia sectorial sobre las mejores prácticas de gestión ambiental, los indicadores sectoriales de comportamiento ambiental y los parámetros comparativos de excelencia" para un conjunto de sectores económicos. Con estas tres Decisiones se aprueban dichos documentos para a los sectores de la administración pública, la fabricación de automóviles y la fabricación de aparatos electrónicos y eléctricos:

- Decisión (UE) 2019/61 de la Comisión, de 19 de diciembre de 2018, relativa al documento de referencia sectorial sobre las mejores prácticas de gestión ambiental, los indicadores sectoriales de comportamiento ambiental y los parámetros comparativos de excelencia para el sector de la administración pública en el marco del Reglamento (CE) n. ${ }^{\circ} 1221 / 2009$, relativo a la participación voluntaria de organizaciones en un sistema comunitario de gestión y auditoría medioambientales (EMAS).

- Decisión (UE) 2019/62 de la Comisión, de 19 de diciembre de 2018, relativa al documento de referencia sectorial sobre las mejores prácticas de gestión medioambiental, los indicadores sectoriales de comportamiento medioambiental y los parámetros comparativos de excelencia para el sector de la fabricación de automóviles en el marco del Reglamento (CE) n. 1221/2009, relativo a la participación voluntaria de organizaciones en un sistema comunitario de gestión y auditoría medioambientales (EMAS). 
- Decisión (UE) 2019/63 de la Comisión, de 19 de diciembre de 2018, relativa al documento de referencia sectorial sobre las mejores prácticas de gestión medioambiental, los indicadores sectoriales de comportamiento medioambiental y los parámetros comparativos de excelencia para el sector de la fabricación de aparatos eléctricos y electrónicos en el marco del Reglamento (CE) n. ${ }^{\circ}$ 1221/2009 del Parlamento Europeo y del Consejo, relativo a la participación voluntaria de organizaciones en un sistema comunitario de gestión y auditoría medioambientales (EMAS).

Durante el periodo comprendido en esta crónica también se han adoptado dos Decisiones referentes a la etiqueta ecológica:

- Decisión (UE) 2019/418 de la Comisión, de 13 de marzo de 2019, por la que se modifican las Decisiones (UE) 2017/1214, (UE) 2017/1215, (UE) 2017/1216, (UE) 2017/1217, (UE) 2017/1218 y (UE) 2017/1219 [notificada con el número C(2019) 1851]. Establece excepciones a la aplicación de ciertos criterios para la concesión de la etiqueta ecológica a algunos productos.

- Decisión (UE) 2019/1134 de la Comisión, de 1 de julio de 2019, por la que se modifican las Decisiones 2009/300/CE y (UE) 2015/2099 en lo que respecta al período de validez de los criterios ecológicos para la concesión de la etiqueta ecológica de la UE a determinados productos, así como de los requisitos de evaluación y comprobación correspondientes [notificada con el número $\mathrm{C}$ (2019) 4626]. Establece la prolongación del periodo de validez de la etiqueta ecológica para ciertos productos.

Debido a la inminente retirada de Reino Unido de la Unión Europea, se aprobó la Decisión (UE) 2019/504 del Parlamento Europeo y del Consejo, de 19 de marzo de 2019, por la que se modifica la Directiva 2012/27/UE relativa a la eficiencia energética y el Reglamento (UE) 2018/1999 sobre la gobernanza de la Unión de la Energía y de la Acción por el Clima, a causa de la retirada del Reino Unido de Gran Bretaña e Irlanda del Norte de la Unión. Esta permite la actualización de los objetivos de la Unión sobre consumo de energía sin tener en cuenta Reino Unido. Tal i como establece el Reglamento (UE) 2018/1999, dichos objetivos para 2030 son el $32,2 \%$ de energía primaria, de manera que 
los "1273 millones de toneladas equivalentes de petróleo (Mtep) y el consumo de energía final de 956 Mtep" se deben adaptar a una UE sin el Reino Unido.

Por último, se aprobó la Decisión (UE) 2018/881 del Consejo, de 18 de junio de 2018, por la que se insta a la Comisión a que presente un estudio acerca de las opciones de las que dispone la Unión para dar respuesta a las conclusiones del Comité de Cumplimiento del Convenio de Aarhus en relación con el asunto ACCC/C/2008/32 y, si resulta adecuado a la luz de los resultados del estudio, una propuesta de Reglamento del Parlamento Europeo y del Consejo por el que se modifique el Reglamento (CE) n.॰1367/2006. El Convenio sobre el acceso a la información, la participación del público en la toma de decisiones y el acceso a la justicia en materia de medio ambiente (o Convenio de Arhus), viene acompañado de un Comité de Cumplimiento, que en 2017 realizó un estudio sobre el acceso a la justicia ambiental en la Unión. La presente Decisión pretende dar respuesta al incumplimiento del Convenio por parte de la legislación europea que el informe puso de manifiesto.

\subsection{Actos delegados}

a) Reglamentos delegados

El Registro de la Unión de derechos de emisión ha sido objeto de tres Reglamentos Delegados durante el periodo comprendido en esta crónica. Los dos primeros hacen una actualización de las funciones del Registro a la luz de la nueva legislación en materia de comercio de emisiones de $\mathrm{CO}^{2}$ :

- Reglamento Delegado (UE) 2019/1122 de la Comisión, de 12 de marzo de 2019, que completa la Directiva 2003/87/CE del Parlamento Europeo y del Consejo en lo que respecta al funcionamiento del Registro de la Unión

- Reglamento Delegado (UE) 2019/1124 de la Comisión, de 13 de marzo de 2019, por el que se modifica el Reglamento Delegado (UE) 2019/1122 en lo que respecta al funcionamiento del Registro de la Unión a efectos del Reglamento (UE) 2018/842 del Parlamento Europeo y del Consejo

El tercero, el Reglamento Delegado (UE) 2019/1123 de la Comisión, de 12 de marzo de 2019, por el que se modifica el Reglamento (UE) n. ${ }^{\circ}$ 389/2013 en lo relativo a la ejecución técnica del segundo período de compromiso del 
Protocolo de Kioto, establece un proceso de compensación de las emisiones positivas para Noruega i Liechenstain, países que participan del régimen de comercio de derechos de emisión de la Unión pero que no son parte del acuerdo de cumplimiento conjunto durante el segundo período de compromiso del Protocolo de Kioto, que finaliza el 31 de diciembre de 2020. Dicha compensación se realizará mediante inscripción en el Registro de la Unión.

En el ámbito del transporte se aprobaron cuatro reglamentos delegados:

- Reglamento Delegado (UE) 2019/839 de la Comisión, de 7 de marzo de 2019, por el que se modifica el Reglamento (UE) n. $540 / 2014$ del Parlamento Europeo y del Consejo sobre el nivel sonoro de los vehículos de motor y de los sistemas silenciadores de recambio. Establece la prohibición de la inclusión de sistemas de desconexión de los sistemas de aviso acústico de los vehículos híbridos y eléctricos por una cuestión de seguridad de los usuarios de la vía pública.

- Reglamento Delegado (UE) 2019/986 de la Comisión, de 7 de marzo de 2019, por el que se modifican los anexos I y II del Reglamento (UE) n. ${ }^{\circ}$ 510/2011 del Parlamento Europeo y del Consejo en lo que se refiere al seguimiento de las emisiones de $\mathrm{CO} 2$ de los vehículos comerciales ligeros nuevos homologados en un proceso multifásico. Establece como metodología para calcular el nivel de emisiones de $\mathrm{CO}^{2}$ de los vehículos comerciales ligeros el método de interpolación aprobado por el Reglamento (UE) 2017/1151. Son los fabricantes mismos quienes deben informar a la Comisión de dichos niveles, así como de los datos utilizados para su cálculo.

- Reglamento Delegado (UE) 2019/888 de la Comisión, de 13 de marzo de 2019, por el que se modifica el anexo I del Reglamento (UE) 2018/956 del Parlamento Europeo y del Consejo, en lo que atañe a los datos sobre los vehículos pesados nuevos que deben ser objeto de seguimiento y comunicación por parte de los Estados miembros y los fabricantes. Establece especificaciones sobre los datos que los Estados miembros deben registrar respecto las emisiones de $\mathrm{CO}^{2}$ de los vehículos pesados de nueva fabricación.

- Reglamento Delegado (UE) 2019/897 de la Comisión, de 12 de marzo de 2019, por el que se modifica el Reglamento (UE) n. ${ }^{\circ}$ 748/2012 incluyendo en el 
anexo I la verificación de la conformidad basada en el riesgo y la aplicación de los requisitos de protección medioambiental. Entre otros cambios que no interesan a la presente crónica, este Reglamento delegado modifica el Reglamento (UE) 748/2012 para que incluya los requisitos de protección medioambiental del anexo 16 del Convenio sobre Aviación Civil Internacional.

En el ámbito agrícola, aunque fuertemente vinculado al de la energía y el transporte, se aprobó el Reglamento Delegado (UE) 2019/807 de la Comisión, de 13 de marzo de 2019, por el que se completa la Directiva (UE) 2018/2001 del Parlamento Europeo y del Consejo en lo que respecta a la determinación de las materias primas con riesgo elevado de provocar un cambio indirecto del uso de la tierra de cuya superficie de producción se observa una expansión significativa a tierras con elevadas reservas de carbono y la certificación de los biocarburantes, los biolíquidos y los combustibles de biomasa con bajo riesgo de provocar un cambio indirecto del uso de la tierra. La Comisión estaba obligada a su elaboración en virtud de la Directiva (UE) 2018/2001. Su importancia radica en la constatación que ciertos usos de la tierra pueden contrarrestar los efectos positivos de la reducción de emisiones de gases de efecto invernadero por del uso de biocarburantes.

En el ámbito de la pesca se aprobaron dos Reglamentos Delegados relativos a la obligación de desembarque de ciertas pesquerías demersales:

- Reglamento Delegado (UE) 2019/905 de la Comisión, de 13 de marzo de 2019, que modifica el Reglamento Delegado (UE) 2018/2034 de la Comisión, por el que se establece un plan de descartes para determinadas pesquerías demersales en aguas noroccidentales para el período 2019-2021

- Reglamento Delegado (UE) 2019/906 de la Comisión, de 13 de marzo de 2019, que modifica el Reglamento Delegado (UE) 2018/2035, por el que se detallan las disposiciones de aplicación de la obligación de desembarque en determinadas pesquerías demersales del mar del Norte para el período 20192021. 
b) Decisiones delegadas

En el periodo de que se ocupa esta crónica se aprobaron dos Decisiones delegadas. En primer lugar y en el ámbito de las emisiones de gases de efecto invernadero, encontramos la Decisión Delegada (UE) 2019/708 de la Comisión, de 15 de febrero de 2019, que completa la Directiva 2003/87/CE del Parlamento Europeo y del Consejo en lo referente a la determinación de los sectores y subsectores que se consideran en riesgo de fuga de carbono para el período 2021-2030.

En segundo lugar, en el ámbito pesquero se adoptó la Decisión Delegada (UE) 2019/910 de la Comisión, de 13 de marzo de 2019, por la que se establece el Programa Plurianual de la Unión para la Recopilación y la Gestión de Datos Biológicos, Medioambientales, Técnicos y Socioeconómicos en los Sectores de la Pesca y la Acuicultura. Este programa plurianual comprende el periodo 2020-2021 y establece los requisitos de los datos que los Estados miembros deben reportar relacionados con el sector de la pesca.

\subsection{Actos de ejecución}

\section{a) Reglamentos de ejecución}

En el periodo analizado en esta crónica se aprobaron dos Reglamentos de ejecución relativos a los hidrofluorocarburos:

- Reglamento de Ejecución (UE) 2019/522 de la Comisión, de 27 de marzo de 2019, por el que se modifica el Reglamento de Ejecución (UE) n. ${ }^{\circ}$ 1191/2014, en lo que atañe a la notificación de los datos relativos a la producción, importación y exportación de polioles que contengan hidrofluorocarburos con arreglo al artículo 19 del Reglamento (UE) n. ${ }^{\circ}$ 517/2014.

- Reglamento de Ejecución (UE) 2019/661 de la Comisión, de 25 de abril de 2019, por el que se garantiza el buen funcionamiento del registro electrónico de las cuotas de comercialización de hidrofluorocarburos. Este fija los requisitos de la información que las empresas que comercializan hidrofluorocarburos deben inscribir en el registro electrónico creado a tal efecto. 
En el sector del transporte se aprobó el Reglamento de Ejecución (UE) 2019/987 de la Comisión, de 29 de mayo de 2019, por el que se modifica el Reglamento de Ejecución (UE) n. ${ }^{\circ}$ 293/2012 en lo que se refiere al seguimiento de las emisiones de $\mathrm{CO}^{2}$ de los vehículos comerciales ligeros nuevos homologados en un procedimiento multifásico. Establece una obligación de información por vía electrónica a los fabricantes de vehículos ligeros sobre las emisiones de $\mathrm{CO}^{2}$ de los nuevos vehículos.

El Reglamento de Ejecución (UE) 2019/1177 de la Comisión, de 10 de julio de 2019, que modifica el Reglamento (UE) n. ${ }^{\circ} 142 / 2011$ en lo relativo a las importaciones de gelatina, subproductos aromatizantes y grasas extraídas, por su parte, determina ciertas modificaciones al anexo del Reglamento (UE) 142/2011 que modifican los requisitos que han de cumplir ciertos productos para su importación.

También se aprobó el Reglamento de Ejecución (UE) 2019/1262 de la Comisión, de 25 de julio de 2019, por el que se modifica el Reglamento de Ejecución (UE) 2016/1141 con el fin de actualizar la lista de especies exóticas invasoras preocupantes para la Unión. Añade diecisiete especies invasoras preocupantes más a la lista ya establecida en el Reglamento de Ejecución (UE) 2016/1141.

El Reglamento de Ejecución (UE) 2019/1397 de la Comisión, de 6 de agosto de 2019, relativo a los requisitos de diseño, construcción y rendimiento y a las normas de ensayo para equipos marinos y por el que se deroga el Reglamento de Ejecución (UE) 2018/773, por su lado, actualiza la lista de equipos marinos sujetos a requisitos armonizados de la Unión en base a los instrumentos internacionales a que hace referencia la Directiva 2014/90/UE.

En el ámbito de la pesca se aprobaron cuatro Reglamentos de ejecución:

- Reglamento de Ejecución (UE) 2019/479 de la Comisión, de 22 de marzo de 2019, por el que se efectúan deducciones de las cuotas de pesca disponibles para determinadas poblaciones en 2018 a causa de la sobrepesca practicada en años anteriores en otras poblaciones y por el que se modifica el Reglamento de Ejecución (UE) 2018/1969 
- Reglamento de Ejecución (UE) 2019/1190 de la Comisión, de 11 de julio de 2019, por el que se modifica el Reglamento de Ejecución (UE) n. ${ }^{\circ}$ 185/2013 con respecto a deducciones de las cuotas de pesca asignadas a España para 2019

- Reglamento de Ejecución (UE) 2019/662 de la Comisión, de 25 de abril de 2019, por el que se prorroga la excepción a lo dispuesto en el Reglamento (CE) n. ${ }^{\circ}$ 1967/2006 del Consejo en lo concerniente a la distancia mínima de la costa y la profundidad marina mínima en el caso de las redes de tiro desde embarcación para la pesca de chanquete (Aphia minuta), cabotí (Pseudaphya ferreri) y caramel (Spicara smaris) en determinadas aguas territoriales de España (Illes Balears)

- Reglamento de Ejecución (UE) 2019/1248 de la Comisión, de 22 de julio de 2019, por el que se establecen medidas para atenuar una amenaza grave para la conservación de la población de bacalao (Gadus morhua) del Báltico oriental.

\section{b) Directivas de ejecución}

En el periodo de tiempo y ámbito temático relevante para esta crónica solo se aprobó una Directiva de Ejecución, la Directiva de Ejecución (UE) 2019/523 de la Comisión, de 21 de marzo de 2019, por la que se modifican los anexos I a V de la Directiva 2000/29/CE del Consejo, relativa a las medidas de protección contra la introducción en la Comunidad de organismos nocivos para los vegetales o productos vegetales y contra su propagación en el interior de la Comunidad. Tiene por objetivo proteger la Unión de la introducción de plagas externas que puedan introducirse por medio del comercio. Consiste en una modificación del anexo de la Directiva 2000/29/CE del Consejo que debe de ser aplicada por los Estados miembros a partir del 1 de diciembre de 2019.

\section{c) Decisiones de ejecución}

Durante el periodo de tiempo comentado en esta crónica se aprobaron tres Decisiones de Ejecución sobre el cálculo i reducción de las emisiones de $\mathrm{CO} 2$ :

- Decisión de Ejecución (UE) 2019/582 de la Comisión, de 3 de abril de 2019, por la que se confirma o modifica el cálculo provisional de las emisiones 
medias específicas de $\mathrm{CO}^{2}$ y los objetivos de emisiones específicas de los fabricantes de vehículos comerciales ligeros nuevos correspondientes al año natural 2017 y de la agrupación Volkswagen y sus miembros correspondientes a los años naturales 2014, 2015 y 2016, en aplicación del Reglamento (UE) n. ${ }^{\circ}$ 510/2011 del Parlamento Europeo y del Consejo [notificada con el número $\mathrm{C}(2019)$ 2342].

- Decisión de Ejecución (UE) 2019/583 de la Comisión, de 3 de abril de 2019, por la que se confirma o modifica el cálculo provisional de las emisiones medias específicas de $\mathrm{CO}^{2}$ y los objetivos de emisiones específicas de los fabricantes de turismos correspondientes al año natural 2017 y de determinados fabricantes de la agrupación Volkswagen correspondientes a los años naturales 2014, 2015 y 2016, en aplicación del Reglamento (CE) n. ${ }^{\circ}$ 443/2009 del Parlamento Europeo y del Consejo [notificada con el número C(2019) 2359].

- Decisión de Ejecución (UE) 2019/1119 de la Comisión, de 28 de junio de 2019, relativa a la aprobación de una tecnología de iluminación eficiente para el exterior de los vehículos que utiliza diodos emisores de luz para su uso en vehículos de motor de combustión interna y en vehículos eléctricos híbridos no recargables desde el exterior como tecnología innovadora para la reducción de las emisiones de $\mathrm{CO}^{2}$ de los turismos de conformidad con el Reglamento (CE) n. ${ }^{\circ} 443 / 2009$ del Parlamento Europeo y del Consejo.

En relación a la elaboración y comunicación de los datos sobre el tratamiento de residuos por parte de los Estados miembros, se aprobaron dos Decisiones de Ejecución:

- Decisión de Ejecución (UE) 2019/665 de la Comisión, de 17 de abril de 2019, que modifica la Decisión 2005/270/CE, por la que se establecen los formatos relativos al sistema de bases de datos de conformidad con la Directiva 94/62/CE del Parlamento Europeo y del Consejo, relativa a los envases y residuos de envases [notificada con el número C (2019) 2805].

- Decisión de Ejecución (UE) 2019/1004 de la Comisión, de 7 de junio de 2019, por la que se establecen normas relativas al cálculo, la verificación y la comunicación de datos sobre residuos de conformidad con la Directiva 
2008/98/CE del Parlamento Europeo y del Consejo y por la que se deroga la Decisión de Ejecución C (2012) 2384 de la Comisión [notificada con el número C(2019) 4114].

En el ámbito de la protección de las aguas contra la contaminación por nitratos de uso agrícola se aprobaron dos Decisiones de Ejecución:

- Decisión de Ejecución (UE) 2019/1205 de la Comisión, de 12 de julio de 2019, por la que se concede la exención solicitada por Bélgica para la región de Flandes, de conformidad con la Directiva 91/676/CEE del Consejo, relativa a la protección de las aguas contra la contaminación producida por nitratos procedentes de fuentes agrarias [notificada con el número C (2019) 5180].

- Decisión de Ejecución (UE) 2019/1325 de la Comisión, de 27 de mayo de 2019, por la que se concede la exención solicitada por el Reino Unido para Irlanda del Norte de conformidad con la Directiva 91/676/CEE del Consejo relativa a la protección de las aguas contra la contaminación producida por nitratos utilizados en la agricultura [notificada con el numero C (2019) 3816].

También en el ámbito de la regulación contra la contaminación, aunque esta vez por contaminación derivada del reciclado de buques, se aprobó la Decisión de Ejecución (UE) 2019/995 de la Comisión, de 17 de junio de 2019, por la que se modifica la Decisión de Ejecución (UE) 2016/2323 por la que se establece la lista europea de instalaciones de reciclado de buques con arreglo al Reglamento (UE) n. ${ }^{\circ}$ 1257/2013 del Parlamento Europeo y del Consejo.

En relación a la producción del aceite de palma se aprobó la Decisión de Ejecución (UE) 2019/1175 de la Comisión, de 9 de julio de 2019, sobre el reconocimiento del régimen voluntario «Roundtable on Sustainable Palm Oil RED» para demostrar el cumplimiento de los criterios de sostenibilidad de conformidad con las Directivas 98/70/CE y 2009/28/CE del Parlamento Europeo y del Consejo.

Por último, en el ámbito de la regulación sobre sustancias peligrosas para el medio ambiente se aprobó la Decisión de Ejecución (UE) 2019/1194 de la Comisión, de 5 de julio de 2019, relativa a la identificación del 4-terc-butilfenol (PTBP) como sustancia extremadamente preocupante con arreglo al artículo 
57, letra f), del Reglamento (CE) n. ${ }^{\circ}$ 1907/2006 del Parlamento Europeo y del Consejo [notificada con el número C (2019) 4987].

\subsection{Acuerdos internacionales y posiciones en organismos internacionales}

Durante el periodo de tiempo objeto de esta crónica se han aprobado dos Decisiones relativas al Convenio de Estocolmo sobre contaminantes orgánicos persistentes:

- Decisión (UE) 2019/448 del Consejo, de 18 de marzo de 2019, relativa a la presentación, en nombre de la Unión Europea, de una propuesta de inclusión del metoxicloro en el anexo $\mathrm{A}$ del Convenio de Estocolmo sobre contaminantes orgánicos persistentes.

- Decisión (UE) 2019/639 del Consejo, de 15 de abril de 2019, por la que se establece la posición que se ha de adoptar, en nombre de la Unión Europea, en la novena reunión de la Conferencia de las Partes respecto a las enmiendas de los anexos A y B del Convenio de Estocolmo sobre contaminantes orgánicos persistentes. En ella se acuerda proponer la inclusión de ciertas sustancias en el Anexo A del Convenio y el endurecimiento de las restricciones a ciertos contaminantes.

También se aprobó la Decisión (UE) 2019/638 del Consejo, de 15 de abril de 2019, relativa a la posición que debe adoptarse en nombre de la Unión Europea en la decimocuarta reunión de la Conferencia de las Partes en relación con determinadas enmiendas a los anexos II, VIII y IX del Convenio de Basilea sobre el Control de los Movimientos Transfronterizos de los Desechos Peligrosos y su Eliminación. Determina una posición favorable a la aprobación de la entrada de deshechos plásticos no peligrosos en el espacio EEE propuesta por Noruega.

Asimismo, se aprobó la Decisión (UE) 2019/1581 del Consejo, de 16 de septiembre de 2019, sobre la presentación, en nombre de la Unión Europea, de propuestas de modificación de los Apéndices I y II de la Convención sobre la Conservación de las Especies Migratorias de Animales Silvestres (CMS), con motivo de la decimotercera reunión de la Conferencia de las Partes. En ella se 
fija la propuesta de añadir una especie en el Apéndice I y otra en el Apéndice II de la CMS.

Durante el periodo analizado se han aprobado nueve Decisiones relativas a la fijación de una posición común en organizaciones internacionales relacionadas con la protección de determinadas especies de peces y otros animales marinos, así como de ecosistemas marinos en sentido amplio:

- Decisión (UE) 2019/812 del Consejo, de 14 de mayo de 2019, relativa a la posición que debe adoptarse, en nombre de la Unión Europea, en la Comisión Interamericana del Atún Tropical (CIAT) y en la Reunión de las Partes en el Acuerdo relativo al programa internacional para la conservación de los delfines, y por la que se deroga la Decisión de 12 de junio de 2014 sobre la posición que debe adoptarse, en nombre de la Unión, en la CIAT.

- Decisión (UE) 2019/824 del Consejo, de 14 de mayo de 2019, relativa a la posición que debe adoptarse, en nombre de la Unión Europea, en la Comisión ampliada del Convenio para la Conservación del Atún Rojo del Sur (Convenio CSBT) y por la que se deroga la Decisión de 12 de junio de 2014 sobre la posición que debe adoptarse, en nombre de la Unión, en la CCSBT.

- Decisión (UE) 2019/860 del Consejo, de 14 de mayo de 2019, relativa a la posición que debe adoptarse, en nombre de la Unión Europea, en la Comisión del Atún para el Océano Índico (CAOI) y por la que se deroga la Decisión de 19 de mayo de 2014 sobre la posición que debe adoptarse, en nombre de la Unión, en la CAOI.

- Decisión (UE) 2019/864 del Consejo, de 14 de mayo de 2019, relativa a la posición que debe adoptarse, en nombre de la Unión Europea, en la Organización para la Conservación del Salmón del Atlántico Norte (NASCO) y por la que se deroga la Decisión de 26 de mayo de 2014 sobre la posición que debe adoptarse, en nombre de la Unión, en la NASCO.

- Decisión (UE) 2019/866 del Consejo, de 14 de mayo de 2019, relativa a la posición que debe adoptarse, en nombre de la Unión Europea, en la conferencia anual de las Partes en la Convención sobre la conservación y ordenación de las poblaciones de abadejos en la región central del mar de 
Bering y por la que se deroga la Decisión de 12 de junio de 2017 por la que se establece la posición que debe adoptarse, en nombre de la Unión, en dicha conferencia anual.

- Decisión (UE) 2019/867 del Consejo, de 14 de mayo de 2019, relativa a la posición que debe adoptarse, en nombre de la Unión Europea, en la Comisión para la Conservación de los Recursos Vivos Marinos Antárticos (CCRVMA) y por la que se deroga la Decisión de 24 de junio de 2014 sobre la posición que debe adoptarse, en nombre de la Unión, en la CCRVMA.

- Decisión (UE) 2019/868 del Consejo, de 14 de mayo de 2019, relativa a la posición que debe adoptarse, en nombre de la Unión Europea, en la Comisión Internacional para la Conservación del Atún del Atlántico (CICAA) y por la que se deroga la Decisión de 8 de julio de 2014 sobre la posición que debe adoptarse, en nombre de la Unión, en la CICAA.

- Decisión (UE) 2019/937 del Consejo, de 27 de mayo de 2019, relativa a la posición que debe adoptarse, en nombre de la Unión Europea, en el marco del Convenio para la conservación del salmón en el Atlántico Norte por lo que refiere a la solicitud de adhesión del Reino Unido a dicho Convenio.

- Decisión (UE) 2019/862 del Consejo, de 14 de mayo de 2019, relativa a la posición que debe adoptarse, en nombre de la Unión Europea, en la Comisión de Pesca del Pacífico Occidental y Central (CPPOC) y por la que se deroga la Decisión de 12 de junio de 2014 sobre la posición que se debe adoptar, en nombre de la Unión, en la CPPOC para la Conservación y Ordenación de las Poblaciones de Peces Altamente Migratorios.

En relación a la participación en organizaciones internacionales relacionadas con la prevención de la contaminación del mar, se aprobó la Decisión (UE) 2019/851 del Consejo, de 14 de mayo de 2019, relativa a la posición que debe adoptarse, en nombre de la Unión Europea, en la Organización Marítima Internacional en el transcurso del $74 .^{\circ}$ período de sesiones del Comité de Protección del Medio Marino y el $1011^{\circ}$ período de sesiones del Comité de Seguridad Marítima respecto de la adopción de enmiendas al anexo II del Convenio internacional para prevenir la contaminación por los buques, enmiendas al Código internacional sobre el programa mejorado de 
inspecciones durante los reconocimientos de graneleros y petroleros, de 2011, enmiendas al Código internacional de dispositivos de salvamento, enmiendas a los formularios C, E y $\mathrm{P}$ del apéndice del Convenio Internacional para la Seguridad de la Vida Humana en el Mar y enmiendas al Código internacional de seguridad para los buques que utilicen gases $u$ otros combustibles de bajo punto de inflamación.

Asimismo, se han aprobado nueve Decisiones para la fijación de una posición común de los miembros de la UE en diversas organizaciones regionales del ámbito pesquero:

- Decisión (UE) 2019/407 del Consejo, de 4 de marzo de 2019, relativa a la celebración, en nombre de la Unión Europea, del Acuerdo para impedir la pesca no reglamentada en alta mar en el Océano Ártico central.

- Decisión (UE) 2019/858 del Consejo, de 14 de mayo de 2019, relativa a la posición que debe adoptarse, en nombre de la Unión Europea, en la reunión de las Partes en el Acuerdo de Pesca para el Océano Índico Meridional (SIOFA) y por la que se deroga la Decisión de 12 de junio de 2017 por la que se establece la posición que debe adoptarse, en nombre de la Unión, en la reunión de las Partes en el SIOFA.

- Decisión (UE) 2019/859 del Consejo, de 14 de mayo de 2019, relativa a la posición que debe adoptarse, en nombre de la Unión Europea, en la Organización Regional de Ordenación Pesquera del Pacífico Sur (SPRFMO) y por la que se deroga la Decisión de 12 de junio de 2017 por la que se establece la posición que debe adoptarse, en nombre de la Unión, en la SPRFMO.

- Decisión (UE) 2019/861 del Consejo, de 14 de mayo de 2019, relativa a la posición que debe adoptarse, en nombre de la Unión Europea, en la Organización de la Pesca del Atlántico Suroriental (SEAFO) y por la que se deroga la Decisión de 12 de junio de 2014 sobre la posición que debe adoptarse, en nombre de la Unión, en la SEAFO.

- Decisión (UE) 2019/863 del Consejo, de 14 de mayo de 2019, relativa a la posición que debe adoptarse, en nombre de la Unión Europea, en la 
Organización de Pesquerías del Atlántico Noroeste (NAFO) y por la que se deroga la Decisión de 26 de mayo de 2014 sobre la posición que debe adoptarse, en nombre de la Unión, en la NAFO.

- Decisión (UE) 2019/869 del Consejo, de 14 de mayo de 2019, relativa a la posición que debe adoptarse, en nombre de la Unión Europea, en la Comisión General de Pesca del Mediterráneo (CGPM) y por la que se deroga la Decisión de 19 de mayo de 2014 sobre la posición que debe adoptarse, en nombre de la Unión, en la CGPM.

- Decisión (UE) 2019/865 del Consejo, de 14 de mayo de 2019, relativa a la posición que debe adoptarse, en nombre de la Unión Europea, en la Comisión de Pesquerías del Atlántico del Nordeste (CPANE) y por la que se deroga la Decisión de 26 de mayo de 2014 sobre la posición que debe adoptarse, en nombre de la Unión, en la CPANE.

- Decisión (UE) 2019/1563 del Consejo, de 16 de septiembre de 2019, relativa a la posición que ha de adoptarse, en nombre de la Unión Europea, en la Comisión de Pesca para el Atlántico Centro-Occidental (Copaco).

- Decisión (UE) 2019/1570 del Consejo, de 16 de septiembre de 2019, relativa a la posición que ha de adoptarse, en nombre de la Unión Europea, en el Comité de Pesca para el Atlántico Centro-Oriental (CPACO).

Durante el periodo cubierto por esta crónica se aprobaron cinco Decisiones relativas a la celebración o aplicación del protocolo asociado a diversos acuerdos bilaterales de pesca con terceros países:

- Decisión (UE) 2019/1332 del Consejo, de 25 de junio de 2019, relativa a la firma, en nombre de la Unión, y a la aplicación provisional del Acuerdo de Colaboración de Pesca Sostenible entre la Unión Europea y la República de Gambia y del Protocolo de aplicación de dicho Acuerdo de Colaboración.

- Decisión (UE) 2019/1088 del Consejo, de 6 de junio de 2019, relativa a la firma, en nombre de la Unión Europea, y a la aplicación provisional del Protocolo de aplicación del Acuerdo de Colaboración en el Sector Pesquero entre la Comunidad Europea y la República de Guinea-Bisáu (2019-2024). 
- Decisión (UE) 2019/951 del Consejo, de 17de mayo de 2019, relativa a la firma, en nombre de la Unión Europea, y a la aplicación provisional del Protocolo de aplicación del Acuerdo de Asociación en el Sector Pesquero entre la Comunidad Europea y la República de Cabo Verde (2019-2024).

- Decisión (UE) 2019/441 del Consejo, de 4 de marzo de 2019, relativa a la celebración del Acuerdo de colaboración de pesca sostenible entre la Unión Europea y el Reino de Marruecos, de su Protocolo de aplicación y del Canje de Notas adjunto al Acuerdo.

- Decisión (UE) 2019/385 del Consejo, de 4 de marzo de 2019, sobre la celebración del Protocolo relativo a la aplicación del Acuerdo de Asociación en el sector pesquero entre la Unión Europea y la República de Costa de Marfil (2018-2024).

Por último, cabe mencionar aquí que la salida del Reino Unido motivó la aprobación de la Decisión (UE) 2019/510 del Consejo, de 25 de marzo de 2019, relativa a la posición que debe adoptarse, en nombre de la Unión Europea, en el marco del Convenio sobre la futura cooperación multilateral en los caladeros del Atlántico Nororiental por lo que se refiere a la solicitud de adhesión del Reino Unido a dicho Convenio. Mediante esta Decisión, la UE aprueba la adhesión de Reino Unido al Convenio como parte autónoma. Se pretende evitar los efectos negativos para todas las partes implicadas de la posible inefectividad sobrevenida del Convenio debido a la retirada de la Unión por parte del Reino Unido.

\section{OTRAS ACTIVIDADES Y ACTOS EN EL ÁMBITO DE LA POLÍTICA EUROPEA DEL MEDIO AMBIENTE}

\subsection{Consultas públicas}

Durante el periodo de tiempo recogido en esta crónica se iniciaron ocho consultas públicas europeas. En el ámbito del control y reducción de las emisiones de $\mathrm{CO}^{2}$ encontramos tres de ellas. En primer lugar la "Consultation on the list of candidate Projects of Common Interest in cross-border carbon dioxide transport infrastructure", abierta del 18 de marzo de 2019 al 9 de junio de 2019, pretende recabar opiniones de las múltiples partes interesadas para 
determinar qué proyectos de infraestructuras de transporte transfronterizo de $\mathrm{CO}^{2}$ deben ser declarados como proyectos de interés común por la Unión Europea.

En segundo lugar, el 27 de mayo de 2019 se abrió a la participación la consulta pública "Industrial emissions - evaluating the EU rules", que se cerró el cuatro de septiembre de 2019. Esta tenía por objetivo obtener el parecer de las partes interesadas para hacer una evaluación del funcionamiento de la Directiva sobre emisiones industriales.

Por último, entre el 11 de septiembre y el 6 de noviembre de 2019, estuvo abierta la consulta pública "Energy - European Partnership for clean hydrogen (Horizon Europe programme)". Esta se dirigía a la comunidad investigadora, y en especial a anteriores participantes del Programa Marco de Investigación 2014-2020, con el objetivo de recabar opiniones sobre los partenariados institucionales europeos propuestos por la Comisión bajo el programa Horizonte Europa.

El sector del transporte en relación al medio ambiente ha generado durante el periodo analizado hasta tres consultas públicas. Entre el 6 de agosto de 2019 y el 29 de octubre de 2019 estuvo abierta la consulta pública "End-of-life vehicles - evaluating the EU rules". La Directiva 2000/53/EC trata de minimizar el impacto medioambiental de los vehículos en la fase final de su vida. Debido a la obligación de evaluar esta directiva a finales de 2020, la Comisión consulta a las partes interesadas en el tratamiento de estos vehículos.

Entre el 11 de septiembre de 2019 y el 4 de diciembre de 2019 estuvo abierta la consulta pública "Urban Mobility in the EU“. Esta tenía por objetivo preguntar sobre el impacto del Paquete de Movilidad Urbana, que tenía como uno de sus objetivos principales la reducción de las emisiones de CO2.

Por último, encontramos la consulta pública "European Partnership for Clean Aviation", que estuvo abierta a la participación entre el 30 de julio de 2019 y el 27 de agosto de 2019. Se dirigía a la comunidad científica por tal de valorar la inclusión en el programa europeo de investigación Horizonte Europa de propuestas dirigidas al desarrollo de tecnologías de aviación de bajas emisiones. 
Entre el 13 de junio de 2019 y el 8 de agosto de 2019 estuvo abierta la consulta pública "Sustainable batteries - EU requirements". Esta tenía por objetivo obtener información por parte de todas las partes interesadas para la elaboración de una regulación europea sobre baterías que establezca estándares comunes para la fabricación de baterías sostenibles.

Finalmente, en el ámbito de la política pesquera también se abrió una consulta pública durante el periodo de tiempo que interesa a esta crónica. Entre el 11 de junio de 2019 y el 20 de agosto e 2019 se pudo participar en la consulta "Posibilidades de pesca para 2020 en virtud de la política pesquera común. Con esto se pretendía permitir a la ciudadanía europea dar su opinión sobre la forma en que se establecen las cuotas de pesca en la política pesquera común.

\subsection{Resoluciones no legislativas del Parlamento Europeo}

Durante el periodo de tiempo comprendido en esta crónica el Parlamento europeo aprobó tres resoluciones no legislativas relevantes desde el punto de vista medio ambiental.

- Resolución del Parlamento Europeo, de 1 de marzo de 2018, sobre las perspectivas y desafíos para el sector apícola de la Unión (2017/2115(INI)). En ella se reconoce el papel vital que las abejas realizan en la polinización de las flores y la necesidad de su protección. El Parlamento Europeo pide a la Comisión que incremente y facilite el acceso del sector apícola en la política agraria común, tanto por lo que atañe a la financiación como a la simplificación administrativa. Es más, sugiere a la Comisión que estudie dedicar un régimen de ayudas para los apicultores a partir de 2020 que tenga en cuenta la función ecológica de las abejas.

- Resolución del Parlamento Europeo, de 13 de marzo de 2018, sobre el papel de las regiones y las ciudades de la UE en la aplicación del Acuerdo de París de la CP 21 sobre el cambio climático (2017/2006(INI)). En ella el Parlamento anima a la Comisión a reforzar la financiación para la consecución de los objetivos climáticos en el Marco Financiero Plurianual para el periodo 20212027 y ampliar la proporción del gasto respecto al periodo anterior. Asimismo, hace un llamamiento a los Estados miembros a hacer una mejor aplicación del 
principio de subsidiariedad y que permitan a las ciudades y municipios participar de forma más decidida en la lucha contra el cambio climático.

- El mismo día que la resolución anterior, se aprobó la Resolución del Parlamento Europeo, de 13 de marzo de 2018, sobre una estrategia europea sobre los sistemas de transporte inteligentes cooperativos (2017/2067(INI)). El Parlamento Europeo pretende dar así un soporte político al desarrollo de sistemas de transporte sostenibles cooperativos por su por su potencialidad de reducción de gases de efecto invernadero por parte del sector del transporte entre otras razones. La resolución insta a la Comisión a establecer un calendario con objetivos específicos para 2029 para el desarrollo de estos sistemas, así como que junto a los Estados miembros mantenga un financiamiento para su investigación.

\subsection{Informes y comunicaciones de la Comisión Europea}

Durante el periodo analizado en esta crónica la Comisión Europea ha emitido hasta once informes sobre temas medioambientales. Algunos de ellos vienen acompañados por documentos de trabajo del personal de la Comisión. De estos podemos enmarcar en el ámbito de la evaluación de las políticas ambientales de la Unión a tres de ellos:

- Informe de la Comisión al Parlamento Europeo, al Consejo, al Comité Económico y Social Europeo y al Comité de las Regiones sobre la evaluación del VII Programa de Acción en materia de Medio Ambiente. Este viene acompañado del "Commission Staff Working Document: Evaluation of the 7th Environment Action Programme to 2020 'Living well, within the limits of our planet' Accompanying the document Report from the Commission to the European Parliament, the Council, the Europe Economic and Social Committee and the Committee of the Regions on the evaluation of the 7th Environment Action Programme".

- Informe de la Comisión al Parlamento Europeo, al Consejo, al Comité Económico y Social Europeo y al Comité de las Regiones: Revisión de los avances en la aplicación de la estrategia sobre la infraestructura verde de la UE. Viene acompañado del "Commission Staff Working Document: Additional information on the review of implementation of the green infrastructure strategy 
Accompanying the document Report from the commission to the European Parliament, the Council, the European and Economic and Social Committee and the Committee of Regions: Review of progress on implementation of the EU green infrastructure strategy".

- Informe de la Comisión al Parlamento Europeo, al Consejo, al Comité Económico y Social Europeo y al Comité de las Regiones sobre la aplicación del Plan de acción para la economía circular. Viene acompañado del "Commission Staff Working Document Accompanying the document Report from the commission to the European Parliament, the Council, the European and Economic and Social Committee and the Committee of Regions on the implementation of the Circular Economy Action Plan".

En el ámbito de la energía hay que señalar dos Informes:

- Informe de la Comisión al Parlamento Europeo y al Consejo: Evaluación de 2018, con arreglo al artículo 24, apartado 3, de la Directiva 2012/27/UE relativa a la eficiencia energética, de los avances realizados por los Estados miembros en la consecución de los objetivos nacionales de eficiencia energética para 2020 y en la aplicación de las disposiciones de dicha Directiva.

- Informe de la Comisión al Parlamento Europeo, al Consejo, al Comité Económico y Social Europeo y al Comité de las Regiones: Informe de situación en materia de energías renovables.

En relación a la protección del medio marino cabe destacar dos Informes:

- Informe de la Comisión: Informe anual sobre la seguridad de las operaciones relativas al petróleo y al gas mar adentro en la Unión Europea para el año 2017.

- Informe conjunto al Parlamento Europeo y al Consejo: Mejora de la gobernanza internacional de los océanos - Dos años de avances. Viene acompañado del "Joint Commission Staff Working Document Accompanying the document Joint report to the European Parliament and the Council: Improving International Ocean Governance - Two years of progress".

Por último, encontramos cuatro Informes de diversa índole relacionada con el medio ambiente: 
- Informe de la Comisión al Parlamento Europeo, al Consejo, al Comité Económico y Social Europeo y al Comité de las Regiones: Conclusiones del control de adecuación de la legislación más pertinente en materia de sustancias químicas (excepto el REACH) y retos, carencias e insuficiencias que se han identificado. Viene acompañado del "Commission Staff Working Document Fitness Check of the most relevant chemicals legislation (excluding $\mathrm{REACH}$ ), as well as related aspects of legislation applied to downstream industries Accompanying the document Report from the commission to the European Parliament, the Council, the European and Economic and Social Committee and the Committee of Regions: Findings of the Fitness Check of the most relevant chemicals legislation (excluding $\mathrm{REACH}$ ) and identified challenges, gaps and weaknesses".

- Informe de la Comisión al Parlamento Europeo, al Consejo, al Comité Económico y Social Europeo y al Comité de las Regiones sobre el estado de la expansión de la producción de cultivos alimentarios y forrajeros pertinentes en todo el mundo.

- Informe de la Comisión al Parlamento Europeo, al Consejo, al Comité Económico y Social Europeo y al Comité de las Regiones sobre la aplicación y el impacto en el medio ambiente y en el funcionamiento del mercado interior de la Directiva 2006/66/CE del Parlamento Europeo y del Consejo, de 6 de septiembre de 2006, relativa a las pilas y acumuladores y a los residuos de pilas y acumuladores y por la que se deroga la Directiva 91/157/CEE.

- Informe de la Comisión al Parlamento Europeo, al Consejo, al Comité Económico y Social Europeo y al Comité de las Regiones y al Banco Europeo de Inversiones sobre la ejecución del Plan de acción estratégico para las baterías: creación de una cadena de valor estratégica para las baterías en Europa.

Asimismo, la Comisión realizó un total de cuatro Comunicaciones, dos de las cuales también vienen acompañadas de documentos de trabajo del personal de la Comisión:

- Comunicación de la Comisión al Parlamento Europeo, al Consejo, al Comité Económico y Social Europeo y al Comité de las Regiones: Unidos para 
contribuir a la Unión de la Energía y a la Acción por el Clima. Establecimiento de las bases para el éxito de la transición hacia una energía limpia. Viene acompañado del "Commission Staff Working Document: Assessment of the national forestry accounting plans regulation (EU) 2018/841 of the European Parliament and of the Council on the inclusion of greenhouse gas emissions and removals from land use, land use change and forestry in the 2030 climate and energy framework, and amending Regulation(EU)No 525/2013 and Decision No 529/2013/EU Accompanying the document Communication from the Commission to the European Parliament, the Council, the European Economic and Social Commitee and the Committee of the Regions United in delivering the Energy Union and Climate Action - Setting the foundations for a successful clean energy transition". También acompaña esta Comunicación el "Commission Staff Working Document Accompanying the document Communication from the Commission to the European Parliament, the Council, the European Economic and Social Committee and the Committee of the Regions: United in delivering the Energy Union and Climate Action - Setting the foundations for a successful clean energy transition".

- Comunicación de la Comisión al Parlamento Europeo y al Consejo relativa a la Cumbre sobre la Acción Climática de 2019, organizada en Nueva York, bajo los auspicios del Secretario General de las Naciones Unidas.

- Comunicación de la Comisión al Parlamento Europeo, al Consejo, al Comité Económico y Social Europeo y al Comité de las Regiones: Intensificar la actuación de la UE para proteger y restaurar los bosques del mundo. Viene acompañado del "Commission Staff Working Document Accompanying the document Communication from the Commission to the European Parliament, the Council, the European Economic and Social Committee and the Committee of the Regions: Stepping up EU Action to Protect and Restore the World's Forests".

- Comunicación de la Comisión al Parlamento Europeo, al Consejo y al Comité Económico y Social Europeo: Enfoque estratégico de la Unión Europea en materia de productos farmacéuticos en el medio ambiente. 


\subsection{Comunicaciones conjuntas de la Comisión Europea y el Alto Representante}

Cabe mencionar aquí la elaboración conjunta entre la Comisión y el Alto Representante de tres Comunicaciones que, aunque se circunscriben al ámbito de la acción exterior de la Unión Europea, son relevantes a efectos de esta crónica por contener muchas referencias a aspectos medioambientales que también integran las relaciones internacionales con otros países o regiones:

- Comunicación conjunta al Parlamento Europeo, el Consejo Europeo y el Consejo: UE-China - Una perspectiva estratégica. Esta Comunicación señala la necesidad de cooperar con China por unas finanzas más sostenibles medioambientalmente y para una inversión en los Balcanes Occidentales por parte de la China que respete los estándares medioambientales europeos.

- Comunicación conjunta al Parlamento Europeo y al Consejo: La Unión Europea, América Latina y el Caribe: aunar fuerzas para un futuro común. En relación a los países de América Latina y Caribe se destaca la importancia de la cooperación medioambiental como elementos centrales para la cohesión social y el fortalecimiento de la sociedad civil organizada en los bloques de "asociación en aras de la democracia" y "asociación en aras de la resiliencia". No obstante, se echa en falta un énfasis mayor en la importancia del medioambiente en la cooperación económica, que se aborda en el primer bloque "asociación en aras de la prosperidad".

- Comunicación conjunta al Parlamento Europeo y al Consejo: La UE y Asia Central: nuevas oportunidades para una asociación más estrecha. Se destaca la importancia del medioambiente, el cambio climático y el agua como elementos centrales para la resiliencia de las sociedades de la región. En ese sentido se propone al Consejo y al Parlamento Europeo seguir apoyando tanto políticamente, como de forma técnica y económica, a los países centroasiáticos desde un enfoque regional.

\subsection{Dictámenes del Comité de las Regiones y del Comité Económico y Social Europeo}


El Comité de las Regiones emitió cuatro Dictámenes temáticamente relevantes para esta crónica:

- Dictamen del Comité de las Regiones sobre «Hacia un octavo Programa de Acción en materia de Medio Ambiente (PMA)».

- Dictamen del Comité Europeo de las Regiones sobre el «Plan de Acción: Financiar el desarrollo sostenible».

- Dictamen del Comité Europeo de las Regiones sobre la «Propuesta de Reglamento del Parlamento Europeo y del Consejo relativo a los requisitos mínimos para la reutilización del agua».

- Dictamen del Comité Europeo de las Regiones - Modelos de asunción local en materia de energía y el papel de las comunidades locales de energía en la transición energética en Europa.

El Comité Económico y Social Europeo, a su vez, emitió diez dictámenes pertinentes para esta crónica:

- Dictamen del Comité Económico y Social Europeo sobre la comunicación de la Comisión al Parlamento Europeo, al Consejo Europeo, al Consejo, al Comité Económico y Social Europeo, al Comité de las Regiones y al Banco Europeo de Inversiones «Un planeta limpio para todos - La visión estratégica europea a largo plazo de una economía próspera, moderna, competitiva y climáticamente neutra [COM (2018) 773 final].

- Dictamen del Comité Económico y Social Europeo sobre la propuesta de Reglamento del Parlamento Europeo y del Consejo por el que se modifica el Reglamento (UE) 2015/757 con objeto de tomar en debida consideración el sistema mundial de recopilación de datos sobre el consumo de fueloil de los buques [COM (2019) 38 final-2019/0017(COD)].

- Dictamen del Comité Económico y Social Europeo sobre la Comunicación de la Comisión al Parlamento Europeo, al Consejo, al Comité Económico y Social Europeo y al Comité de las Regiones - Una bioeconomía sostenible para Europa: consolidar la conexión entre la economía, la sociedad y el medio ambiente [COM (2018) 673 final]. 
- Dictamen del Comité Económico y Social Europeo sobre la «Comunicación de la Comisión al Parlamento Europeo, al Consejo, al Comité Económico y Social Europeo y al Comité de las Regiones - Hacia un marco de la Unión Europea más exhaustivo en materia de alteradores endocrinos» [COM (2018) 734 final].

- Dictamen del Comité Económico y Social Europeo sobre la propuesta de Decisión del Parlamento Europeo y del Consejo por la que se adaptan la Directiva 2012/27/UE del Parlamento Europeo y del Consejo, relativa a la eficiencia energética [modificada por la Directiva (UE) 2018/2002], y el Reglamento (UE) 2018/1999 del Parlamento Europeo y del Consejo [relativo a la gobernanza de la Unión de la Energía], con motivo de la retirada del Reino Unido de la Unión Europea [COM(2018) 744 final - 2018/0385 (COD)].

- Dictamen del Comité Económico y Social Europeo sobre la propuesta de Reglamento del Parlamento Europeo y del Consejo relativo a la adaptación de las obligaciones de notificación en el ámbito de la política de medio ambiente y por el que se modifican las Directivas 86/278/CEE, 2002/49/CE, 2004/35/CE, 2007/2/CE, 2009/147/CE y 2010/63/UE, los Reglamentos (CE) n. ${ }^{\circ}$ 166/2006 y (UE) n. ${ }^{\circ}$ 995/2010, y los Reglamentos (CE) n. ${ }^{\circ} 338 / 97$ y (CE) n. ${ }^{\circ} 2173 / 2005$ del Consejo [COM(2018) 381 final - 2018/0205 (COD)].

- Dictamen del Comité Económico y Social Europeo sobre la propuesta de Reglamento del Parlamento Europeo y del Consejo relativo a los requisitos mínimos para la reutilización del agua (programa evolutivo) [COM (2018) 337 final].

- Dictamen del Comité Económico y Social Europeo sobre la «Comunicación de la Comisión al Parlamento Europeo, al Consejo, al Comité Económico y Social Europeo y al Comité de las Regiones - Una Europa que protege: Aire puro para todos» [COM (2018) 330 final].

- Dictamen del Comité Económico y Social Europeo sobre la propuesta de Reglamento del Parlamento Europeo y del Consejo por el que se establecen medidas de conservación y control aplicables en la zona de regulación de la Organización de Pesquerías del Atlántico Noroeste y por el que se derogan los Reglamentos (CE) 2115/2005 y (CE) 1386/2007 del Consejo [COM (2018) 577 final - 2018/0304 (COD)]. 
- Dictamen del Comité Económico y Social Europeo sobre la «Propuesta de Reglamento del Parlamento Europeo y del Consejo por el que se modifica el Reglamento (CE) n. ${ }^{\circ} 1224 / 2009$ del Consejo, y se modifican los Reglamentos (CE) n. ${ }^{\circ}$ 768/2005, (CE) n. ${ }^{\circ}$ 1967/2006 y (CE) n. ${ }^{\circ}$ 1005/2008 del Consejo y el Reglamento (UE) 2016/1139 del Parlamento Europeo y del Consejo en lo que respecta al control de la pesca» [COM (2018) 368 final - 2018/0193 (COD)].

\section{PROCEDIMIENTOS DE INFRACCIÓN}

Durante el periodo de tiempo cubierto por esta crónica la Comisión decidió llevar al Tribunal de Justicia de la Unión Europea (TJUE) a siete Estados miembros por infracciones de la normativa europea medioambiental. El 7 de marzo de 2019 lo hizo con Italia y Chipre, mientras que el 25 de julio lo hizo con Suecia. La Comisión considera que Italia ha estado infringiendo la normativa en lo que atañe al tratamiento de aguas urbanas durante trece años en dieciséis regiones italianas. Asimismo, la Comisión cree que Chipre no está cumpliendo con esta normativa en 31 aglomeraciones urbanas y denuncia que las autoridades chipriotas no prevén hacerlo antes de 2029. Suecia, por su parte, no ha aplicado la normativa de tratamiento de aguas urbanas en 6 poblaciones que deberían haberlo hecho desde 2005.

Otro ámbito de la normativa que ha generado litigiosidad durante los siete meses analizados aquí es la relativa a la prevención de la polución del aire regulada en la Directiva 2008/50/EC. Italia fue llevada el 7 de marzo ante el TJUE también por este motivo, a parte de la inaplicación de la normativa de tratamiento de aguas urbanas que comentábamos antes. Según la Comisión a día de hoy Italia no ha conseguido respetar los valores de calidad del aire acordados para 2010 en 10 aglomeraciones de población habitadas por 7 millones de personas. En segundo lugar, también por incumplimiento de los estándares de calidad del aire, la Comisión llevó el 25 de julio de 2019 a Bulgaria y a España ante el TJUE. En el caso de Bulgaria los incumplimientos se dan en la parte sud-este del país, donde se encuentran las cuatro mayores centrales termoeléctricas del país. En España los límites se rebasan en Madrid, Barcelona, y las áreas del Vallès y Baix Llobregat. 
Por último, hay que mencionar que Grecia fue llevada ante el TJUE el 25 de julio de 2019 por parte de la Comisión Europea por su incumplimiento del deber de proteger ciertos hábitats y especies de animales. En Grecia se encuentran un conjunto de hábitats designados por la red europea Natura 2000 sobre los cuales la Comisión argumenta que Grecia debería haber establecido medidas de conservación de acuerdo con la Directiva 92/43/EEC. En 2012 expiró el plazo para el establecimiento de tales medidas y objetivos. 